\title{
Plateforme d'étude et de conception de systèmes de radiocommunications
}

\author{
Jacques VERDIER, Guillaume VILLEMAUD, Jean-Marie GORCE \\ Départements Télécommunications et Génie Electrique de l'INSA de LYON \\ 20, avenue Albert Einsteïn 69621 Villeurbanne cedex - France \\ jacques.verdier@insa-lyon.fr
}

\section{RESUME}

La complexité des systèmes radio actuels comme futurs, couplée à la nécessité pour un étudiant ou un élève ingénieur, issu du domaine de l'Electrical Engineering et/ou des Télécommunications d'accéder à une formation adaptée à son futur métier, impose aux établissements de se doter de moyens matériels et logiciels ambitieux, à la pointe des nouvelles technologies. Les élèves -ingénieurs des départements Génie Electrique et Télécommunications de l'INSA de Lyon disposent maintenant des techniques les plus avancées en conception, modélisation et test de systèmes de radiocommunications. Nous présentons dans cet article une plateforme transversale dédiée à l'étude de ces systèmes qui permet aux élèves d'appréhender les défis majeurs liés aux nouvelles technologies existantes ou futures qui sont axés principalement sur les performances de l'interface radio (bruit, sensibilité, stabilité, cadence d'échantillonnage) et sur les processus algorithmiques (traitement et analyse du signal à une cadence élevée). Deux sujets pratiques destinés aux étudiants de $5^{\text {ème }}$ année (étudiants INSA) et de $2^{\text {nd }}$ année de MASTER (étudiants hors INSA) sont présentés de manière synthétique. Ils traitent, pour le premier, des algorithmes SIMO appliqués aux récepteurs multistandards et pour le second sujet, de l'influence des imperfections des différents blocs radiofréquences d'une liaison radio WLAN (Wireless local area network) $802.11 \mathrm{~g}$ sur les performances globales. Le contenu de ces enseignements sera plus largement développé lors de la conférence.

Mots clés : Radiocommunications, GSM, UMTS, WIFI, Electronique RF et numérique, Traitement du signal

\section{INTRODUCTION}

Au cours des 10 dernières années de nombreuses technologies de transmission sans fil ont vu le jour [1][2]. Ces technologies ont émergé à la fois dans le domaine de la téléphonie mobile (GSM, UMTS) et dans le domaine des réseaux locaux sans fil (WiFi, Hiperlan, Bluetooth). Ce fort développement des technologies de l'information et de la communication (TIC) a naturellement amené les universités et les écoles d'ingénieurs à faire évoluer leur formation type EEA. C'est particulièrement le cas dans ce secteur des communications sans fil où il est demandé des formations plus directement reliées à ce domaine. Il apparaît ainsi des filières spécifiques aux noms évocateurs tels que Electronique des Communications ou encore Systèmes Intégrés pour les Radiocommunications.

Dans ce contexte technologique bouillonnant, les formations de $2^{\text {ème }}$ et $3^{\text {ème }}$ cycle doivent apporter aujourd'hui modules d'enseignement adaptés et réactifs en matière de pédagogie et d'outils logiciel et matériel associés. Ainsi, au niveau de l'INSA de Lyon et plus précisément des départements Télécommunications (TC) et Génie Electrique (GE), nous avons développé, en collaboration avec la société Agilent Technologies, une plateforme de tests et d'étude des systèmes de radiocommunications et de validation des modèles de simulation.

Dans cet article nous présentons cette plateforme ainsi que 2 sujets pratiques que nous proposons aux étudiants de dernières années (INSA TC \& GE ou MASTER_M2).

\section{PLATEFORME D'ETUDE DES SYSTEMES DE RADIOCOMMUNICATION}

Les technologies sans fil se développent, mais un des défis de ces prochaines années concerne l'interaction entre ces technologies. Les constructeurs travaillent sur des récepteurs multi-normes, par exemple GSM-WiFi, permettant à un seul équipement de se connecter à différents réseaux. Les équipements actuels intègrent alors plusieurs interfaces radio. En parallèle, pour ceux qui sont à l'étude, on cherche à développer les techniques favorisant l'implantation entièrement logicielle d'un émetteur-récepteur radio gage d'une véritable reconfigurabilité du système.

La plate-forme radio présentée sur la figure 1 est un outil d'analyse qui permet d'éprouver les techniques les plus avancées en conception, modélisation et test des systèmes de radiocommunications. Tous les maillons d'une chaîne d'émission-réception peuvent ainsi être étudiés, aussi bien les parties électroniques analogiques, numériques et RF (amplificateurs, modulateurs, filtres, antennes, DSP, ...) que les aspects et modèles liés à la propagation (canal radio) et bien entendu les techniques de traitement du signal associées.

Cette plateforme est composée d'équipements industriels performants développés par la société Agilent Technologies [3][4]: le logiciel ADS (Advanced Design System) de CAO de circuits RF et microondes, deux générateurs d'onde arbitraire 
(ESG4438C) et un analyseur de spectre vectoriel (VSA89641) avec 2 entrées RF et des entrées I et Q.

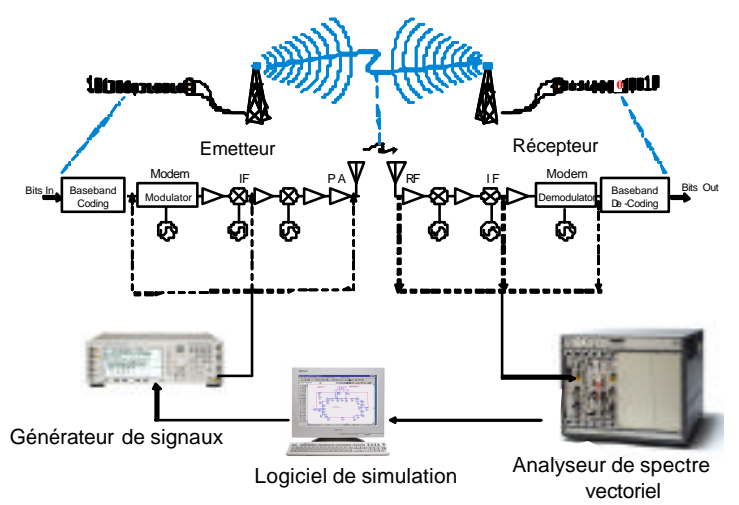

figure 1: Schéma de la plateforme radio. Visualisation des possibilités fréquentielles des études : RF, FI et bande de base

La plateforme présente à ce jour une structure $2 \times 2$ voies qui fonctionne jusqu'à $6 \mathrm{GHz}$ et qui possède, au niveau de la réception, une bande passante de $36 \mathrm{MHz}$. Ses potentialités résident dans la forte interaction matériel-logiciel qui peut permettre les études en RF, comme en FI (fréquence intermédiaire) ou en bande de base. La source d'onde arbitraire est capable de générer n'importe quel signal complexe que l'on peut alors analyser après propagation, dans un environnement réel, avec l'analyseur de spectre vectoriel. Le logiciel d'analyse vectorielle associée peut alors démoduler le signal reçu et offrir différentes options de visualisation comme la densité spectrale de puissance en fonction de la fréquence (le spectre obtenu est alors comparé au spectre d'émission), l'ACPR qui est définie comme le rapport entre la puissance moyenne dans le canal adjacent en fréquence (gauche ou droit) sur la puissance moyenne dans le canal de transmission dans le diagramme de constellation des états, l'EVM (Error Vector Measurement) qui représente les distorsions d'amplitude et de phase sur le diagramme de constellation, le taux d'erreur par bit (TEB)... Généralement, l'optimisation isolée des techniques de transmission mène régulièrement à des résultats décevants si la mise en pratique matérielle n'a pas été prise en compte. L'utilisation de cet outils est alors un atout véritable puisqu'il permet une conception globale d'un système à différents niveaux (hardware, traitement du signal) dans un contexte de fonctionnement réaliste. En effet, un signal radio crée sous ADS peut être chargé dans la mémoire interne du générateur ESG puis émis via une antenne. Le signal reçu et mesuré par l'analyseur VSA peut être enregistré et transféré à nouveau sous le logiciel ADS. Cette interaction matériel-logiciel peut se situer en RF, en FI comme au niveau des voies I et Q ou après le convertisseur Analogique Numérique. Sur la figure 2, nous avons reporté l'exemple où les entrées I et $Q$ de l'analyseur vectoriel sont utilisées permettant d'étudier la qualité du signal (TEB) entre l'entrée « $\mathrm{RF}$ » et la récupération des voies I et $\mathrm{Q}$ du bloc conçu et réalisé.

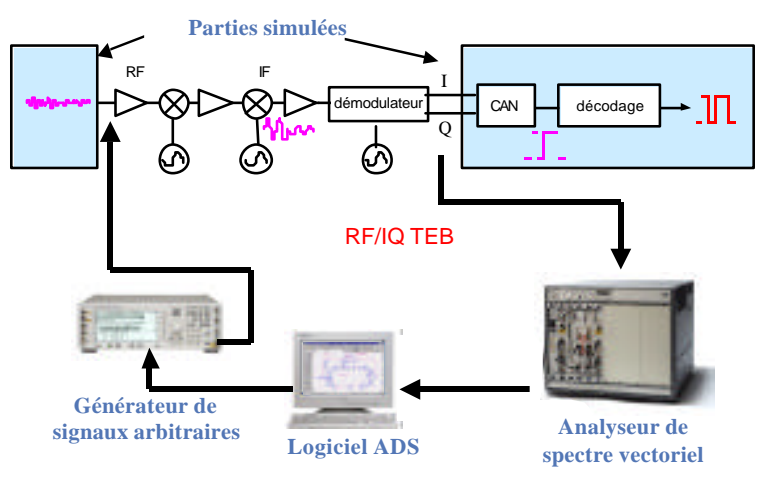

figure 2: Exemple d'étude du «TEB» entre le signal RF et les signaux $\mathrm{I}$ et $\mathrm{Q}$ en sortie du démodulateur.

Dans le récepteur schématisé, l'émetteur et son signal ont été modélisés sous ADS (simulateur Ptolémy) et émis via l'ESG. Au niveau du récepteur, les parties allant de l'antenne aux signaux $\mathrm{I}(\mathrm{t})$ et $\mathrm{Q}(\mathrm{t})$ sont soit des réalisations pour la conception du système ou soit des circuits déjà existants. Le signal de sortie est alors injecté dans le VSA pour être à nouveau traité sous ADS. Sur la figure 3, un second exemple est schématisé où l'on a représenté cette fois -ci la partie émettrice dans laquelle un composant réel de la chaîne est sous test. Via ADS, le signal RF émis par l'ESG comportant les imperfections du modulateur, du mélangeur, du filtre RF... va «attaquer» l'entrée du dispositif «hardware». Le signal de sortie RF est alors re-injecté dans la chaîne via le VSA. Sur la figure 4, nous présentons des résultats obtenus dans le cas particulier (figure 4) où le dispositif à tester est un amplificateur de puissance. Le signal utilisé pour stimuler l'entrée du PA a été créé sous ADS et présente des distorsions en amplitude et un certain niveau d'interférence intersymbol. Le signal d'entrée présente un EVM de $6.7 \%$. Le signal en sortie du PA présente un EVM largement augmenté (8.9 \%) ainsi qu'un forte déformation de son spectre fréquentiel. C'est ce signal, tenant compte des imperfections du PA, qui est injecté dans le reste de la chaîne à étudier.

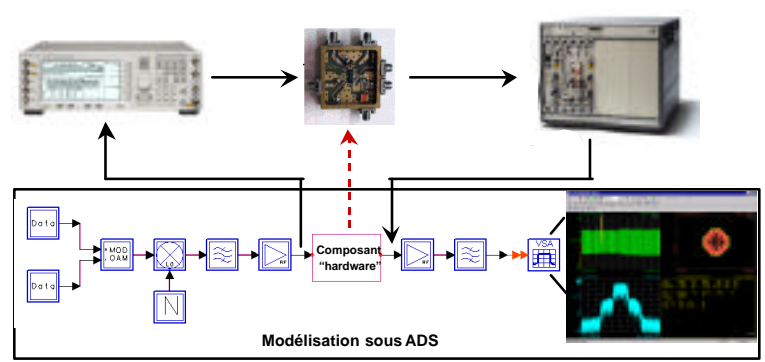

figure 3: Exemple d'aide à la conception/ vérification du système radio.

Enfin, et ce n'est pas le moindre des intérêts de cette plateforme, il est possible, pour accélérer la conception d'un système, de modéliser rapidement sous Ptolemy via des mesures effectuées sur des analyseurs de réseau ou de spectre, sur des bancs de 
caractérisation en puissance, (...) des dispositifs tels que amplificateurs, filtres, oscillateurs qui viennent d'être conçus (mais pas le système dans son ensemble) et de les évaluer dans la chaîne d'Emission-Réception.

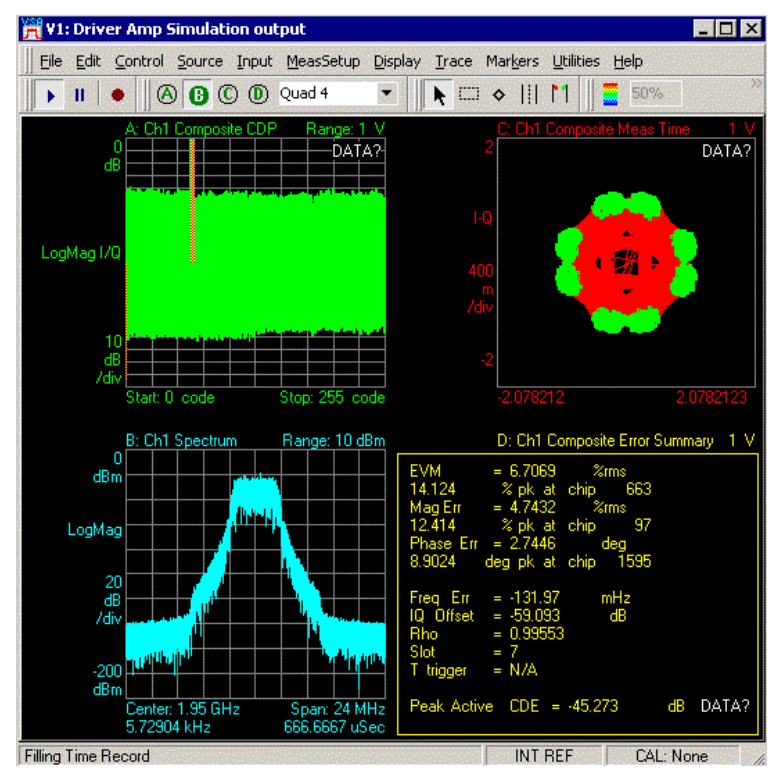

figure 4a : Signaux (spectre, constellation, EVM) à l'entrée de l'amplificateur de puissance sous test.

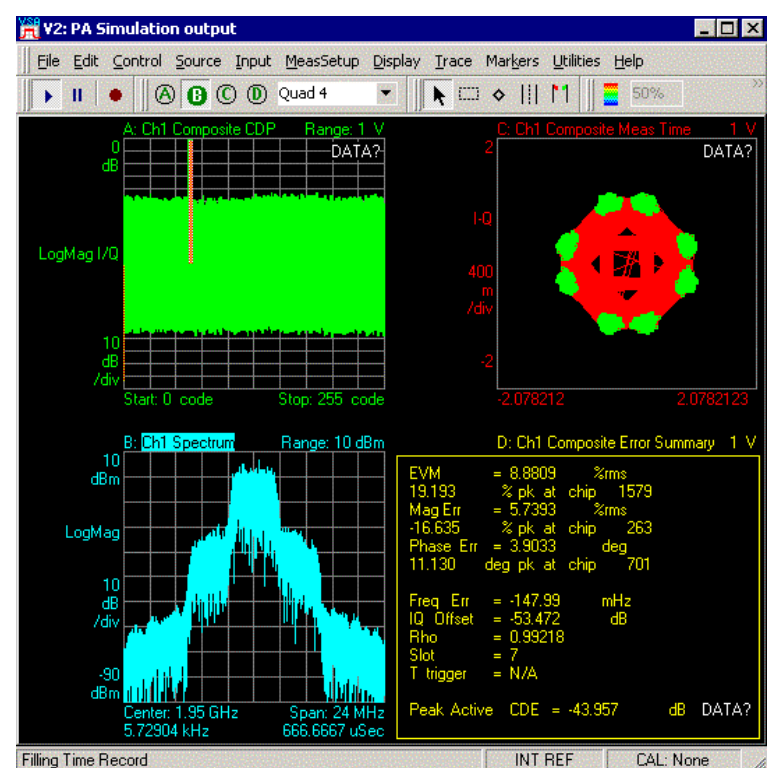

figure 4b : Signaux (spectre, constellation, EVM) à la sortie de l'amplificateur de puissance sous test.

Tous ces exemples montrent clairement les atouts de cette plate-forme transversale véritablement innovante pour les enseignements de type EEA autour des systèmes de radiocommunications. Couplée à d'autres bancs de caractérisation de dispositifs et de circuits, elle peut enrichir les enseignements plus directement liés à la conception de circuits et/ou systèmes entièrement intégrés ou hybrides.

Nous allons maintenant présenter dans les deux parties suivantes deux types d'étude que nous proposons aux étudiants de $5^{\text {ème }}$ année des départements GE et TC ou de MASTER (M2). Le travail demandé s'inscrit dans la continuité des enseignements liés aux radiocommunications de $3^{\text {ème }}$ et $4^{\text {ème }}$ années qui présentent les méthodes de conception et de simulation des différents éléments d'un émetteur/récepteur de radiocommunications numériques, aussi bien les éléments RF que les éléments numériques.

\section{ETUDE D'ALGORITHMES SIMO APPLIQUES AUX RECEPTEURS MULTI- STANDARDS.}

Un grand intérêt de cette plateforme est donc de pouvoir comparer les résultats obtenus avec un canal radio simulé ou mesuré (comme dans l'exemple de la figure 5). De nombreuses techniques permettent de lutter contre les effets néfastes (atténuation, masquage, évanouissements...) de ce canal de propagation. Actuellement, l'ensemble des nouvelles normes de communications proposent d'améliorer les performances de ces liaisons par le recours à des systèmes à antennes multiples. On désigne par MIMO (Multiple Input Multiple Output) un système basé sur plusieurs antennes à l'émission et à la réception. Cette approche suppose une modification de standard à l'émission, alors qu'une approche SIMO (Single Input Multiple Output) uniquement en réception peut s'adapter à toute norme existante en améliorant les performances. Nous allons donc présenter ici une étude de ce type réalisable avec le matériel disponible.

Le principe de traitement SIMO est fondé sur le fait qu'en disposant judicieusement plusieurs antennes sur un même terminal, chacune recevra une copie différente (car n'ayant pas subi les mêmes influences du canal) du signal émis. Dès lors, selon les caractéristiques de la forme d'onde et du type de canal traversé, différents algorithmes des plus simples aux plus sophistiqués peuvent être appliqués pour recombiner ces différentes copies en améliorant au final le signal reçu.

Le travail des étudiants est ici d'étudier différents algorithmes SIMO et de comparer leurs performances via des simulations sous ADS et des campagnes de mesures sur la plateforme d'étude des systèmes de radiocommunications.

\subsection{Traitements SIMO utilisés}

Les structures SIMO développées sont appliquées aux standards $802.11 \mathrm{~b}$ et $802.11 \mathrm{~g}$ qui permettent d'obtenir des débits importants. En fonction de l'environnement et du standard, tous les traitements SIMO n'entraînent pas la même hausse de performances. En effet, ces deux normes WiFi cohabitent dans la même bande de fréquence mais utilisent des formes d'ondes très différentes qui ne réagissent donc pas de la même façon à la traversée de l'environnement. Dans le cas proposé, quel que soit le standard et le type de diversité considérés, les pondérations sont calculées pour minimiser le critère d'erreur quadratique moyenne (en anglais Minimun 
Mean-Square Error ou MMSE) entre le signal recombiné et le signal de référence constitué du champ «PLCP preamble » des trames 802.11 b ou du premier symbole du champ «long preamble»des trames 802.11g [5]. Des chaînes de simulation de récepteur $802.11 \mathrm{~b}$ et $802.11 \mathrm{~b}$ à 4 voies ont été développées sous ADS. Le tableau 1 résume les algorithmes utilisés en fonction de l'environnement et des standards de communication.

\begin{tabular}{|l|c|c|}
\hline & $802.11 \mathrm{~b}$ & $802.11 \mathrm{~g}$ \\
\hline Diversité spatiale & SMI-MMSE & SMI-MMSE \\
\hline Diversité temporelle & RAKE - 2D & $\mathrm{X}$ \\
\hline Diversité fréquentielle & $\mathrm{X}$ & SF - MMSE \\
\hline
\end{tabular}

tableau 1 - Traitements SIMO utilisés.

Les étudiants doivent intégrer que comme les trames $802.11 \mathrm{~b}$ sont construites par étalement de spectre, les techniques basées sur l'utilisation de la diversité temporelle sont judicieuses. D'autre part, la construction des trames $802.11 \mathrm{~g}$ basée sur le principe de modulation OFDM les encourage à profiter de la diversité fréquentielle. Dans le cas d'un traitement SMI (Sample Matrix Inversion, inversion matricielle basée sur une séquence de référence dans le signal), seules des pondérations spatiales sont appliquées. Le traitement Rake 2D (récepteur râteau à 2 dimensions) correspond à une égalisation temporelle sur chaque voie avant recombinaison optimale des 4 signaux reçus. Enfin, le traitement SF-MMSE (minimisation spatio-fréquentielle) permet d'appliquer des pondérations différentes sur chaque voie mais identiques pour les même groupes de sous-porteuses OFDM. Différentes structures de simulations comprenant ces traitements sont fournies et intègrent 4 modélisations de canal indépendantes et regroupant les voies au sein d'un bloc de traitement Matlab. Les étudiants peuvent ainsi cerner les nombreux paramètres à optimiser en fonction du standard et du canal de propagation. On présente ici quelques résultats obtenus en termes de TEB (mais bien entendu les autres critères présentés précédemment peuvent être observés). Plus précisément, les courbes suivantes donnent ce TEB en fonction du rapport entre l'énergie moyenne par bit d'information et la densité spectrale d'un bruit blanc (Eb/No), conduisant les élèves à comprendre l'influence en termes de choix des modulations utilisées.

Les étudiants peuvent ainsi appréhender à la fois les impacts du canal de propagation, mais également le profit que l'on peut tirer de l'utilisation de plusieurs antennes pour lutter contre l'effet le plus néfaste : l'évanouissement. On peut également voir la nécessité de bien dimensionner les structures matérielles ou logicielles des récepteurs en fonction du type de canal (profondeur d'égalisation, taille des séquences de référence, taille des trames en fonction de la cohérence...).

Les figures $5 \mathrm{a}$ et $5 \mathrm{~b}$ présentent les performances simulées obtenues dans le cas d'une transmission 802.11 b à $11 \mathrm{Mbps}$ et $802.11 \mathrm{~g}$ à $36 \mathrm{Mbps}$ à travers le canal de propagation ETSI A $\left(\mathrm{t}_{\mathrm{rms}}=50 \mathrm{~ns}\right.$, étalement temporel de $300 \mathrm{~ns})$. Le traitement de diversité spatiale SMI-MMSE est appliqué à chacun des standards, alors que le traitement SF-MMSE (en groupant les fréquences par 4) n'est utilisé que dans le cas de la transmission $802.11 \mathrm{~g}$.

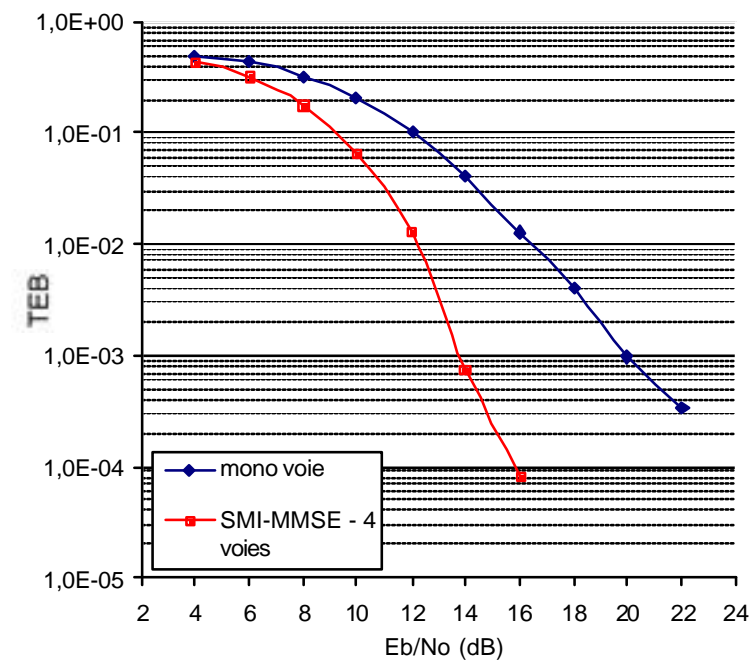

figure 5a: Performances des traitements SIMO pour une transmission 802.11 b.

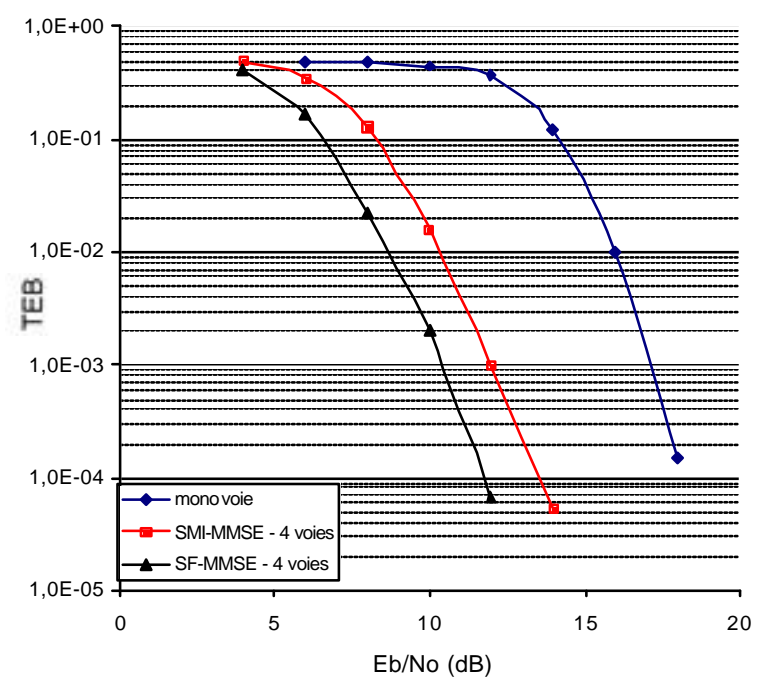

figure 5b : Performances des traitements SIMO pour une transmission $802.11 \mathrm{~g}$.

\subsection{Comparaison simulation-mesure}

Les systèmes ADS développés permettent de simuler différents types de canaux de propagation relatifs à une transmission à $2.4 \mathrm{GHz}$ proposés par le modèle ETSI [6]. Ces canaux sont tous très dispersifs. Ils présentent de nombreux échos et un étalement temporel relativement important. Ils introduisent donc un facteur de diversité très important. De plus, au cours des simulations, comme il n'est pas possible d'introduire les phénomènes de corrélation de voie, ni de couplage entre antennes, il est constaté par les élèves une surévaluation de l'augmentation des performances obtenues grâce aux traitements SIMO. Une évaluation dans un contexte de fonctionnement le plus réel possible (couplage d'antenne, corrélation de canal, canal de propagation plus ou moins dispersif) apparaît clairement nécessaire. 
Des mesures préliminaires sont réalisées grâce à la plateforme radio au sein de nos locaux pour caractériser le canal de propagation qui est celui à notre disposition (banc équivalent à celui de la figure 1, mais avec deux voies de réception sur l'analyseur et deux jeux de mesures pour obtenir 4 acquisitions exploitables). Ce travail permet aux élèves de conclure quant à la fiabilité de certains modèles de canaux. Il est à noter ici, dans la majorité des pièces, que nous disposons d'un canal à étalement temporel beaucoup plus faible que ceux proposés par l'ETSI. Deux environnements de test ont été utilisés : une configuration de transmission proche de l'AWGN, et une configuration de transmission à faible étalement te mporel $\left(\mathrm{t}_{\mathrm{ms}}=48 \mathrm{~ns}\right.$, étalement temporel de $80 \mathrm{~ns}$, bande de cohérence de $5.8 \mathrm{MHz}$ ).

Tous les résultats et analyses comparatives entre mesures et simulations dans différentes configurations seront présentés lors de la conférence.

\section{ETUDE DES LIMITATIONS RF SUR LES PERFORMANCES DU RECEPTEUR 802.11g}

Cette étude, qui s'étale sur un semestre entier, s'inscrit dans la partie dédiée aux éléments RF d'une liaison radio où la conception des circuits analogiques, RF et microondes et le choix de l'architecture (et son dimensionnement) du front-end associé (architectures superhétérodyne, homodyne et faible FI -fréquence intermediaire-) sont au centre de cet enseignement. Pour les élèves-ingénieurs des départements GE et TC de l'INSA, ce module fait suite à l'étude d'algorithmes SIMO précédemment présentée. Il s'agit d'appréhender l'impact des imperfections radiofréquences sur les performances globales d'un récepteur SIMO-OFDM pour le standard 802.11g. En particulier, le travail demandé aux étudiants est d'étudier l'influence du déséquilibre des voies I et $\mathrm{Q}$ en gain et en phase, le bruit de phase de l'oscillateur local ainsi que les non-linéarités de l'amplificateur de puissance utilisé au niveau de l'émetteur. Les performances du système conçu et simulé sous le logiciel ADS sont évaluées en termes de taux d'erreur par bit (TEB), de diagramme de constellation des états pour chaque sous porteuse, et d'évolution de la densité spectrale de puissance du signal. Les résultats présentés concernent des systèmes mono-antenne et quatre antennes (au niveau du récepteur). L'algorithme de traitement est le SMI et deux types de modèle de canaux de propagation, AWGN et multi-trajets, font également partis des conditions de base de cette étude. Dans l'étude proposée, les étudiants ont à leur disposition la chaîne d'émission-réception sous Ptolémy qui est déjà fonctionnelle mais exempt de défauts RF. Ils ont par ailleurs de nombreux articles et ouvrages à leur disposition (dont certains sont reportés ci-après).

Enfin, dans cet article nous nous limitons à l'influence du bruit de phase de l'oscillateur local. Lors de la conférence nous aborderons les autres défauts RF que sont les distorsions harmoniques et d'intermodulation ainsi que le déséquilibre des voies
I et $\mathrm{Q}$ du récepteur.

Il a été montré dans les systèmes mono-antenne que l'influence du bruit de phase d'un oscillateur local sur les performances du récepteur peut être séparée en deux parties [7]. Une contribution dite «erreur de phase du signal» considérée comme du bruit additif sur la phase du signal. Cette erreur est causée par le bruit de phase près de la porteuse. La seconde contribution est liée à une dégradation du bruit dans la bande du signal par mélange du bruit de phase loin de la porteuse avec un signal interférent fort. Pour quantifier ces effets sur le TEB, dans le cas du récepteur $802.11 \mathrm{~g}$, nous faisons développer aux étudiants des modèles de bruit de phase soit via le paramètre PhaseNoiseData de la source N-Tones du simulateur d'ADS-Ptolemy soit des modèles plus élaborés en langage $\mathrm{C}$ (pour des modèles plus physiques intégrant par exemple le modèle de Leeson [1]) permettant de quantifier l'effet des composantes de bruit BF de l'amplificateur intégré dans l'oscillateur (bruit thermique, en 1/f...), leur niveau en $\mathrm{dBc} / \mathrm{Hz}$ ou encore la présence de raies parasites (spurious) en sortie de la PLL (Phase locked loop).

Nous avons reporté sur la figure 6, les performances en terme de TEB en fonction de $\mathrm{Eb} / \mathrm{No}$ dans les cas de récepteur SISO et SIMO-OFDM (1 et 4 antennes) avec un canal AWGN pour modéliser la propagation radio Emetteur-Récepteur. Pour les courbes présentées, c'est le bruit en 1/f qui sert de référence au modèle de bruit de phase de l'oscillateur local comme indiqué ci-après. Nous avons utilisé ici le paramètre «PhaseNoiseData ». PN1, PN2 et PN3 sont les spécifications de l'oscillateur local (OL) en bruit de phase retenues pour ces simulations.

$$
\begin{array}{ll}
\text { PN1 (-? -) : } & \text { pas de bruit de phase } \\
\text { PN2 (-? -) : } & -60 \mathrm{dBc} / \mathrm{Hz} \text { à } 100 \mathrm{~Hz} \\
& -90 \mathrm{dBc} / \mathrm{Hz} \text { à } 1 \mathrm{kHz} \\
& -150 \mathrm{dBc} / \mathrm{Hz} \text { à } 100 \mathrm{kHz} \\
\text { PN3 (- } \Delta-): \quad & -20 \mathrm{dBc} / \mathrm{Hz} \text { à } 100 \mathrm{~Hz} \\
& -50 \mathrm{dBc} / \mathrm{Hz} \text { à } 1 \mathrm{kHz} \\
& -110 \mathrm{dBc} / \mathrm{Hz} \text { à } 100 \mathrm{kHz}
\end{array}
$$

Sur la figure 6 , il est clair que le bruit de phase dégrade sensiblement les performances en taux d'erreur par bit. Pour des niveaux de Eb/No supérieur à $8 \mathrm{~dB}$ on constate une variation d'au moins une décade (de l'ordre de $3.10^{-2}$ avec PN1 et de $3.10^{-1}$ avec $\mathrm{PN} 3$ ) pour le cas $1 \times 4 \mathrm{AWGN}$.

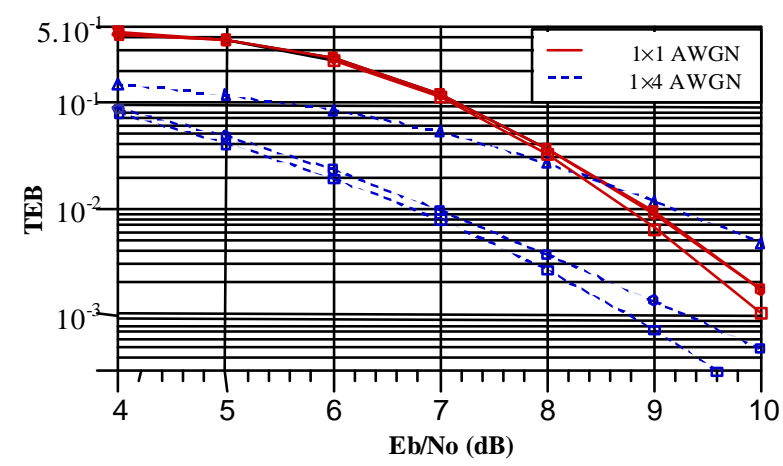

figure 6 : TEB en fonction de Eb/No (canal AWGN) 
Pour pouvoir aller plus loin dans l'analyse, c'est à dire dans la comparaison des récepteurs SISO et SIMO et dans la compensation éventuelle des effets du bruit de phase observés, il est utile de tracer ces résultats en relatif.

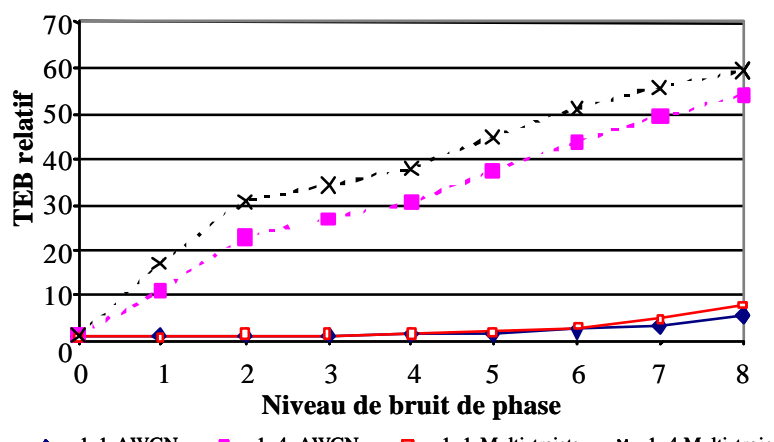

figure 9 : TEB relatif en fonction du niveau de bruit de phase (“0" aucun;"8"-40 dBc/Hz à $100 \mathrm{~Hz}$ )

Sur la figure 9 nous avons reporté cette comparaison en TEB relatif des deux types de récepteur en fonction du niveau de bruit de phase de l'OL et pour les deux modèles de canal: le modèle AWGN et modèle A multi-trajets d'ADS. La référence prise est la valeur de TEB du système 1 antenne sans bruit de phase appliqué. On peut constater que, quel que soit le canal utilisé, cette dégradation des performances est importante et largement augmenté pour le système 4 antennes. En conséquence, l'algorithme SMI n'apparaît pas capable de compenser les effets de l'augmentation du bruit de phase de l'oscillateur local.

Pour conclure sur cette partie, il est important de signaler que le simulateur ADS qui permet de bien quantifier l'impact d'un bruit de phase proche de la porteuse ne permet pas actuellement de travailler à des fréquences d'offset supérieures au $\mathrm{MHz}$ (les résultats observés en simulation sont très variables suivant les paramètres de simulation et donc peu exploitables). Ceci est évidemment préjudiciable à l'étude et à la conception de systèmes OFDM où les problèmes hors bandes peuvent être très présents .

\section{CONCLUSION}

Une approche globale de conception associant l'utilisation d'antennes multiples, la technique d'OFDM et les principes de SDR est un choix reconnu comme réellement judicieux aujourd'hui pour l'analyse et la conception de futurs systèmes sans fils larges bandes. Il apparaît alors clairement aujourd'hui que le vaste domaine des radiocommunications se doit d'être enseigné à nos futurs ingénieurs à hauteur des défis et enjeux de des nouvelles technologies. L'utilisation d'outils logiciels et matériels de l'industrie microélectronique et télécoms est un atout fort et quasi incontournable pour toutes les formations universitaires qui doivent se tourner toujours davantage vers l'international. La plateforme d'étude, de conception et de test des systèmes de radiocommunications que nous venons de présenter répond largement à ces nouveaux besoins de formation. L'interaction matériel-logiciel de cette plate-forme, la possibilité de faire le lien entre les technologies microélectroniques, les composants actifs et passifs, les circuits clés RF ou numériques et le système radio complet d'émissionréception font de cet équipement de formation une innovation importante que l'INSA de Lyon a su développer à l'échelle des deux départements Génie Electrique et Télécommunications c'est à dire pou plus de 200 étudiants par an.

\section{Références bibliographiques}

[1] G. Baudoin, M. Villegas et al “Radiocommunications numériques, tome 1 et 2 ” Ed. Dunod, 623 pages et 446 pages, 2002.

[2] R. Alison "Software Radio: A Modern Approach to Radio Engineering" Ed. Prentice Hall, 592 pages, 2002.

[3] http://eesof.tm.agilent.com/products/ads main.html

[4] P.F. Morlat, H. Parvery, G. Villemaud, J. Verdier, J.M. Gorce, "Global System Evaluation Scheme for Multiple Antennas Adaptive Receivers ", European Conference on Wireless Technology, Manchester, 2006.

[5] P.F. Morlat, P. Mary, G. Villemaud, J.M. Gorce, M. Arndt, "Performance Validation of a MultiStandard and Multi-Antenna Receiver», European Conference on Antennas and Propagation, 2006.

[6] Channel Models for HIPERLAN/2 in different indoor Scenarios. ETSI EP BRAN 3ER1085B, 1998.

[7] S. Wu et al. «Performances analysis on the effect of phase noise in OFDM systems", Proc. IEEE 7th international symposium on Spread Spectrum Techniques and Applications, vol. 1, pp. 133-138, 2002. 